Escuela de Ciencias Sociales y Humanidades, UNED, C.R.

https://revistas.uned.ac.cr/index.php/espiga

ISSN: 1409-4002 • e-ISSN: 2215-454X

\title{
El significado de la feminidad: estudio basado en relatos de vida de mujeres
}

\section{Blanca Luz Sojo-Mora*}

iD https://orcid.org/0000-0002-0488-6799

Recibido: 29 de octubre, 2019 - Aceptado: 20 de febrero, 2020

\section{RESUMEN}

Con el fin de determinar el significado que las características y los roles atribuidos a las mujeres dan a la feminidad, se entrevistó a 14 mujeres, así se ve que el significado de la feminidad proviene de dos factores: apariencia y comportamiento. A partir de esto, se concluye que la feminidad tiene una vinculación directa con el cuerpo de las mujeres y se basa en ideales, estereotipos, roles y normas asignados por la estructura patriarcal, los cuales se han instaurado y permeado en las corporalidades, desde la forma de pensar hasta la reproducción de comportamientos, con el consecuente impacto en la vida de las mujeres. De este modo, se entiende que el modelo de feminidad es socializado por toda una estructura, para ser instaurado, reproducido y mantenido.

Palabras clave: Género, construcción, apariencia, comportamiento.

\section{Formato de citación según APA}

Sojo-Mora, B. (2020). El significado de la feminidad: estudio basado en relatos de vida de mujeres. Revista Espiga, 19 (39), 46-62.

\section{Formato de citación según Chicago-Deusto}

Sojo-Mora, Blanca Luz (2020). «El significado de la feminidad: estudio basado en relatos de vida de mujeres». Revista Espiga 19, n. ${ }^{\circ} 39$ (enero-junio, 2020): 46-62.

* Bachiller en Educación Física, licenciada en Administración Educativa, licenciada en Docencia, máster en Pedagogía con énfasis en diversidad de los procesos educativos y doctora en Estudios de Sociedad y Cultura, Universidad de Costa Rica (UCR). Profesora en la UCR, donde actualmente coordina Vida Estudiantil en la sede del Atlántico y el proyecto Empodero. Áreas de interés: género, mujeres y empoderamiento. Interés de investigación: género. Correo: blanca.sojo@ucr.ac.cr 


\section{Introducción}

\section{Concepto y roles de género}

Según Judith Butler el género es «...el aparato a través del cual tiene lugar la producción y la normalización de lo masculino y lo femenino» ${ }^{1}$; ella asegura que el género podría ser el aparato a través del cual lo femenino y lo masculino se deconstruya y se desnaturalice. Para esta autora, ser femenina no es un hecho natural, sino una representación cultural creada por un conjunto de actos impuestos por el discurso y mantenida por los signos corporales, como los gestos, vestimenta y comportamientos, entre otros. Ella considera el género una construcción basada en los discursos y actuaciones dominantes y no una categoría fija o estable. Así mismo Butler menciona que «el género es performativo (...) siempre es un hacer» ${ }^{2}$, atribuyéndole una condición cambiante.

Otro concepto de género relevante es el acentuado por María Sanahuja, quien lo define como «una construcción cultural y social que determina las expectativas de comportamiento social de las mujeres y hombres y se crea y transmite a través de procesos de socialización de niños y niñas» ${ }^{3}$. En la misma línea conceptual, Celia Vera cita el concepto de género dado por la IV Conferencia Mundial sobre las Mujeres, celebrada en Pekín en setiembre de 1995, asociándolo con una construcción social, histórica y cultural en función de su sexo, «por lo que los roles de género masculino o femenino vienen diferenciados por las funciones, actitudes y capacidades que culturalmente se les atribuye desde el nacimiento a las mujeres y a los hombres» ${ }^{4}$.

Por lo tanto, el género no responde a aspectos naturales o biológicos, sino a aspectos culturales y sociales que establecen lo masculino y lo femenino, los cuales condicionan la orientación sexual y el comportamiento. Todo esto, creado para ser impuesto por medio de discursos y signos corporales hasta ser aceptado como natural.

Al crearse el género y, por consiguiente, la clasificación de lo masculino y lo femenino, también se crean roles de comportamiento para hombres y mujeres que los encierran en la construcción que se ha establecido previamente y no en lo que quisieran ser.

Según Celia Amorós «el género funciona como una adscripción o una apropiación de lo que en una cultura determinada se entiende por femenino o masculino (...) se trata del proceso mediante el cual una representación social es aceptada e incorporada por un individuo como su propia representación $»^{5}$. Kathleen Stassen y Ross Thompson señalan las tres principales teorías sobre los mecanismos de adquisición de los roles masculino y femenino:

1. Judith Butler, Deshacer el género. El género en disputa: el feminismo y la subversión de la identidad (Barcelona: Paidós, 2006), 70.

2. Butler, Deshacer..., 84

3. María Sanahuja, Cuerpos sexuados, objetos y prehistoria (Madrid: Ediciones Cátedra, 2002), 32.

4. Celia Vera, Género, cultura e imagen del cuerpo. En Derecho de las mujeres a su imagen: los trastornos del comportamiento alimentario (anorexia y bulimia) (Universidad de Alicante, 2001), 37.

5. Celia Amorós, Feminismo y filosofía (Madrid: Síntesis, 2000), 275. 
- Teorías psicoanalíticas: la culpa y el miedo son los responsables de que niños y niñas adopten conductas apropiadas al género y el código moral del progenitor del mismo sexo.

- Teorías del aprendizaje social: todos los roles según el género son aprendidos y no innatos, es la sociedad la responsable de enseñarlo consciente o inconscientemente, se dan refuerzos y castigos cuando se comportan de forma que los adultos consideran apropiada o no según su género. Se aprende por medio de la observación.

- Teorías cognitivas: centradas en la comprensión que tienen los pequeños sobre las diferencias de género ${ }^{6}$.

Magdalena León se inclina por la teoría de aprendizaje social. Ella identifica a la sociedad como generadora de la construcción de cualidades distintas (entre) hombre y la mujer, o sea, la construcción de la masculinidad y la feminidad. Esas cualidades se convierten en roles sociales por seguir ${ }^{7}$. Dentro de la sociedad, León ubica a la familia como promotora de los roles de género. Señala que las jerarquías de género son creadas, reproducidas y mantenidas día a día a través de la interacción de los miembros del hogar, «en la familia es donde la división sexual del trabajo, la regulación de la sexualidad y la construcción social y la reproducción de los géneros se encuentra enraizada $»^{8}$. León reafirma que «la adquisición de la masculinidad y la feminidad se da por medio de un aprendizaje social y de conformidad con las normas de un modelo dado»?.

\section{Construcción de lo femenino}

Para Silvia Tubert, la feminidad «no responde a ninguna esencia natural; lo que la mujer parece ser resulta de las ideas y prácticas discursivas sobre la feminidad, que varían su significación en distintas épocas y sociedades» ${ }^{10}$; por lo tanto, no es algo fijo.

La constitución de las identidades femeninas y masculinas está mediada por factores que, más allá de la genética, se relacionan con aspectos psicológicos, sociales y culturales que conforman la identidad. Así, según Purificación Mayobre, «la constitución de la masculinidad y la feminidad es el resultado de un largo proceso, de una construcción, de una urdimbre que se va tejiendo en interacción con el medio familiar y social» ${ }^{11}$.

6. Kathleen Stassen y Ross Thompson, Psicología del desarrollo. Infancia y adolescencia. (Madrid: Médica Panamericana, 1997), 400-405.

7. Magdalena León de Leal, «La familia nuclear: origen de las identidades hegemónicas femenina y masculina», en Género e identidad: ensayos sobre lo femenino y lo masculino, ed. por Gabriela Arango, Magdalena León y Mará Vivero (Bogotá: Ediciones Uniandes, 1995), 179.

8. León, La familia..., 180.

9. Ibíd, 182.

10. Silvia Tubert, «Los ideales culturales de la feminidad y sus efectos sobre el cuerpo de las mujeres», Quaderns de Psicología 12 (2010),161-174, acceso: 03 de marzo, 2020, http://www.quadernsdepsicologia. cat/article/view/760

11. Purificación Mayobre Rodríguez, «La formación de la identidad de género. Una mirada desde la filosofía», Revista venezolana de estudios de la mujer, n. $^{\circ} 12$ (2006): 1, acceso: 03 de marzo, 2020, http://www. scielo.org.ve/scielo.php?script=sci_arttext\&pid=S1316-37012007000100004\&lng=en\&nrm=iso\&tlng=es 
Por su parte, Carmen Caamaño y Ana Rangel indican que «...la feminidad ha estado definida como lo no masculino ${ }^{12}$ conceptualizándolo desde una oposición genérica. Para Amorós, fue gracias a los estudios feministas que se descubrió, desde la historia de la cultura occidental, «lo que se conceptualizaba como femenino y por todas partes surgía la paradoja de un ser definido desde y en función de otros (los varones)» ${ }^{13}$.

La feminidad se puede analizar desde lo superficial pues, para Juan Bautista, la exterioridad es «una característica de lo que podemos llamar posición femenina» ${ }^{14}$. Sin embargo, lo exterior puede ser considerado como uno de los tantos factores desde los que se puede estudiar la feminidad y no el único, porque podría estudiarse desde sus experiencias de vida, su forma de pensar, etc.

Para Santillán, lo femenino se forma a través de las estructuras de poder establecidas por la normatividad sobre los sexos; pero también por la negación a esas normas, por lo cual la autora advierte que la construcción de la feminidad, antes que responder exclusivamente a lo masculino, tiene que ver con las conductas de las mujeres frente a las normas establecidas ${ }^{15}$. En ese sentido, la feminidad puede entenderse como una condición marginal tejida por relaciones de dominación que las mismas mujeres perpetúan desde su posición subordinada.

En la sociedad patriarcal, las mujeres están sujetas al orden jerárquico que les forma, controla y disciplina. Según Doris Fernández, desde que hombres y mujeres nacen, se van «constituyendo como sujetos impregnados de una cultura androcéntrica en la dinámica socialmente determinada, que al mismo tiempo otorga ventajas y poder al hombre» ${ }^{16}$; lo cual, hace que ellas se constituyan desde una posición secundaria y dominada, mientras ellos lo hacen desde una posición hegemónica y dominadora. De esta manera, las potestades para hablar, crear y dominar se asignan a los hombres; mientras a las mujeres, se les asignan roles pasivos, irracionales, sumisos, así como de incompetencia y debilidad.

Para María Venegas, «es a través del cuerpo (...) como tiene lugar la construcción de la identidad o subjetividad corporal femenina, mediante experiencias y prácticas corporales» ${ }^{17}$.

Tubert asegura que las exigencias y modelos asignados a la feminidad intervienen en las manifestaciones de malestar y sufrimiento, la dificultad para lograrlo provoca conflictos con el cuerpo, consigo mismo y con los otros. Agrega que los ideales asociados

12. Carmen Caamaño Morúa y Ana Rangel Núnez, Maternidad, feminidad y muerte (San José: Editorial Universidad de Costa Rica, 2002), 82.

13. Amorós, Feminismo..., 275.

14. Juan Bautista Ritvo, El laberinto de la feminidad y el acto analítico (Buenos Aires: Homo Sapiens, 2009), 9.

15. Santillán, Mujer, deporte y fútbol: una perspectiva de género (Distrito Federal, 2006), 7-9.

16. Doris Fernández Carvajal, «La construcción de la subjetividad de las mujeres en una sociedad Costera», Revista PRAXIS n. ${ }^{\circ} 60$ (2007), 29-48.

17. María del Mar Venegas Medina, «La "mirada normativa del otro", representación del cuerpo femenino y construcción de la identidad corporal a través de la experiencia del cuerpo como espacio de sumisión y resistencia», en Cuerpos de mujeres: miradas, representaciones e identidades, ed. por Gregorio Gil, Carmen, Muñoz Muñoz, Ana y Adelina Sánchez Espinosa (Granada: Editorial Universidad de Granada, 2007), 207. 
con la feminidad no solo son responsables de malestar; sino, también, contribuyen a la subordinación social, legal, económica y familiar, al imponer modelos de identidad ${ }^{18}$.

\section{Desarrollo}

Esta investigación se desarrolló en el marco del Trabajo Final de Graduación «Construcción de las subjetividades femeninas por medio de la práctica de actividades físico deportivas», desarrollada para optar por el grado de doctorado en Estudios de la Sociedad y la Cultura de la Universidad de Costa Rica, presentado y aprobado en el 2015.

Los datos se recolectaron en el 2014 de cinco mujeres que asistían al gimnasio Snake (ubicado en el cantón central de Turrialba), cinco mujeres que asistían al gimnasio Eben Ezer (en el cantón central de Turrialba) y cuatro mujeres que asistían al gimnasio RODDO (en el cantón de Paraíso).

El grupo de estudio corresponde a 14 mujeres, mayores de 18 años y menores de 60 . Se seleccionó la muestra de manera incidental para responder al fácil acceso, a la posibilidad de establecer una buena relación entre la entrevistada y la investigadora, para de este modo generar credibilidad en la información, al interés de la participante, a la comunicación y a su disponibilidad en el factor tiempo para participar de las entrevistas. Por lo tanto, la muestra fue seleccionada de forma no probabilística por conveniencia; para la selección no se usaron criterios estadísticos.

El promedio de edad de las entrevistadas es de 40 años; en el momento de la investigación, siete estaban casadas, una separada, tres en unión libre y tres solteras. Todas trabajaban y son heterosexuales; la mayoría ha cursado estudios universitarios; de ellas, nueve son profesionales, tres terminaron la secundaria y dos la primaria.

Se usó la técnica de la entrevista a profundidad; por medio de la cual se estableció una conversación dirigida hacia los temas de interés para esta investigación; se focalizó de acuerdo con el tema de estudio y sus subtemas; y el objeto por indagar determinó el contenido de las preguntas. No se determinó previamente un número de entrevistas, pues era posible que con algunas de las mujeres se necesitara varias para cumplir el objetivo; sin embargo, todas prefirieron que se les realizara la entrevista en un solo día.

Se procuró un ambiente distendido, informal, cómodo y de confianza para la entrevistada. Se proporcionó un clima atento, respetuoso y sin interrupciones inoportunas; se le dijo que se expresara con sinceridad; también se facilitó y estimuló la expresión de cada una. Además, el uso de grabadora para registrar los detalles de la entrevista se dio con previa autorización, esto con el fin de prestar la suficiente atención a la entrevistada y posibilitar la transcripción sin alteraciones.

Las entrevistas fueron individuales para que la entrevistada fuera la única protagonista, tuviera el tiempo suficiente para responder y no se sintiera intimidada porque las demás escuchen sus respuestas o lo que tenían que responder.

18. Tubert, «Los ideales culturales», 162. 
El momento de la entrevista fue determinado por la entrevistada, según la disponibilidad que tuviera. Las preguntas realizadas ya estaban previamente establecidas en un instrumento que fue guía para la entrevista. Esta guía fue aplicada a tres mujeres con el fin de someterlo a prueba y hacerle mejoras; se eliminaron algunas preguntas, se mejoró la redacción y se agregó preguntas más específicas Una vez realizadas las mejoras, se aplicó a la muestra.

Los pasos seguidos en el proceso de análisis, que permitieron ordenar, categorizar, priorizar e interrelacionar los datos, fueron los siguientes:

1. Realización de las entrevistas.

2. Transcripción.

3. Lectura preliminar de cada texto transcrito.

4. Marcar, señalar y subrayar fragmentos del texto que correspondan a cada subcategoría y categoría de análisis para seleccionarlos. De forma paralela, se hicieron anotaciones sobre narraciones interesantes e importantes.

5. Agrupación de los fragmentos de textos seleccionados por tema de análisis. En la mayoría se cita lo que expresó la entrevistada con sus mismas palabras, con el fin de no alterar la información.

6. Posteriormente, se juntaron todos los fragmentos seleccionados y las anotaciones de una misma entrevista para compararla con las de las otras entrevistas.

7. Una vez integrados todos los fragmentos, se procede a interpretarlos y contrastarlos con la teoría.

Cada uno de estos pasos se siguió con cada entrevista. Además, se procesó las respuestas en tablas de frecuencia con el fin de mostrar la cantidad de respuestas asignadas a un elemento analizado.

\section{Resultados y discusión}

Como parte de los resultados y datos de esta investigación, se incluyen citas de las entrevistas; estas son textuales y fueron seleccionadas por el significado, relevancia y relación con cada tema en mención. Los nombres usados no corresponden a los reales, sino a seudónimos.

\section{El cuerpo controlado para la feminidad}

$\mathrm{Al}$ analizar las entrevistas de forma integral, se determina que el significado de feminidad que ellas asumen proviene, primordialmente, de dos factores. Según la frecuencia de las respuestas, el significado que atribuyen estas mujeres a la feminidad proviene de la apariencia (ropa, apariencia física juvenil, bonita, atractiva, cuidada) y el comportamiento (forma de caminar, de hablar, actitudes de delicadeza, prudencia y tolerancia, gestos). A estos factores se les puede dar el nombre de signos corporales, como los llamó Butler, pues 
se identifica lo femenino como una representación cultural que es mantenida por signos corporales como los gestos, la vestimenta y los comportamientos ${ }^{19}$.

Estos resultados coinciden con la teoría de Bautista, quien asume que la feminidad se puede analizar desde lo externo o superficial ${ }^{20}$. Estas mujeres aluden a signos corporales externos, por lo tanto se puede decir que la feminidad responde a lo visible.

Cada una de ellas asocia la feminidad a diferentes aspectos; la mayoría la asocia con maternidad, belleza, sexualidad, apariencia física, comportamiento y cuerpo. Además, la asocian a la buena figura, juventud, atracción física y clase. La mayoría de estas asociaciones pasan por el cuerpo, por lo que, para ellas, el concepto de feminidad tiene una vinculación directa con el cuerpo de las mujeres, con lo que el cuerpo expresa.

Se puede presumir que la constitución que tienen estas mujeres sobre lo femenino, tiene bases políticas, sociales y culturales, que convergen en sus cuerpos, convirtiéndolos en espacios de tensión. Diversos discursos de poder traspasan el cuerpo y han consolidado un fuerte discurso femenino en el que predominan relaciones no igualitarias entre hombres y mujeres, las cuales giran en torno a un punto de vista androcéntrico que regula el cuerpo de ellas.

Discursos de poder desde la política, ciencia, religión, deporte y medios de comunicación regulan los cuerpos de las mujeres, al controlar la apariencia y el comportamiento. Ante esto, han surgido diversas posiciones feministas acerca del cuerpo femenino. Claudia Mandel brinda un panorama claro sobre tres corrientes feministas fundamentales que se contraponen a discursos existentes:

- Feministas igualitarias, para quienes el cuerpo es un objeto biológico en el que la representación y funcionamiento es político, donde lo femenino, como lo masculino, es determinado socialmente y no de manera biológica.

- Feministas de la construcción social, quienes fundamentan la oposición sexo/género, separando lo biológico (categoría fija o estable) de lo social e ideológico (variable).

- La corriente que asume que el cuerpo no es un objeto ahistórico, acultural y biológicamente dado, sino es un sistema de significación y representación ${ }^{21}$.

\section{Normas del ideal femenino}

Entre todas las informantes, las características que se asocian con una mujer femenina son diferentes; sin embargo, muchas de sus respuestas se relacionan primero con el comportamiento y después con la apariencia. Ellas consideran que una mujer debe caminar y tener gestos suaves y delicados. Además, también al hablar, lo debe hacer de una manera suave; debe ser prudente, tolerante, sensible, amable, sociable, educada, coqueta

\footnotetext{
19. Butler, Deshacer..., 70.

20. Bautista, El laberinto..., 69 .

21. Claudia Mandel, Mapa del cuerpo femenino. Una lectura deconstructiva de creadoras visuales en Costa Rica (Costa Rica: Editorial UCR, 2010), 6-8.
} 
y, además, debe andar el cabello arreglado y largo, maquillada, usar accesorios, vestidos, enaguas y ropa ajustada. Cada una asume diferentes características para una mujer femenina y no solamente una.

Ellas piensan la feminidad desde el estereotipo de la docilidad, sumisión y obediencia. De alguna manera, esta forma de concebirse como mujer refleja el poder ejercido sobre el cuerpo de las mujeres. Para Rita Segato, el cuerpo de las mujeres actualmente ocupa un objetivo estratégico dentro de una guerra no convencional en la que, a pesar de las leyes y políticas públicas de protección para las mujeres, existe vulnerabilidad en los cuerpos femeninos y feminizados, lo cual conduce a la violencia ${ }^{22}$. Según Segato, «La guerra se ha transformado, confiriendo a la escena bélica una nueva estructura y asignando al cuerpo femenino o feminizado un papel nuevo que lo transfiere de una posición marginal a una posición central» ${ }^{23}$.

Porque una mujer feminizada desde la docilidad, sumisión y obediencia es débil, frágil e insegura, con cuerpos y mentes disciplinados ante el patriarcado. Con una visión de mundo en el que lo femenino se doblega ante lo masculino, caracterizado por manifestaciones de dominación desde el poder simbólico, acciones naturalizadas socialmente.

Las siguientes son algunas expresiones sobre el comportamiento que ellas juzgan como aceptables o prohibitivas:

- «No fumar, ni hablar como hombres.» (Nancy)

- «Vocabulario suave, una forma de caminar como mujer, sentarse como mujer.» (Rebeca)

- «Yo no veo una mujer femenina si usa palabras obscenas o una mujer haciendo cosas que se consideran muy masculinas como, por ejemplo, mujeres en competencia de pesas. Una mujer femenina es más medida en sus cosas, en sus palabras, en sus gestos, no se anda exhibiendo.» (Yesenia)

- «No sentarse toda abierta porque no es decente, la manera en cómo se expresa y actúa, no hablar como hombres.» (Irma)

- «Con recato, que no sea grosera.» (Patricia)

- «La manera en que hable una mujer, cómo se exprese; yo siento que hay palabras que no calzan en la boca de un hombre, menos en la boca de una mujer. Los gestos, en la calle no me pueden ver tomando ni fumando. Como mujer no me permito andar haciendo el ridículo.» (Ana)

- «Andar el pelo bien bonito, aplanchado, usar enagua o vestido.» (Nancy)

- «Usar tacón alto, con vestido cortito, arriba de la rodilla (...) debe ser más suave, más esbelta, no tan delgada, delicada, que sea sensible.» (Laura)

22. Rita Laura Segato, «Las nuevas formas de la guerra y el cuerpo de las mujeres», Revista Sociedade e Estado, n. ${ }^{\circ} 29$ (2014), 341-371.

23. Segato, «Las nuevas formas...», 343 . 
- «No verse vulgar porque eso hace que se pierda la feminidad.» (Marta)

- «La forma de desenvolverse, de dirigirse, la forma de hablar, los ademanes que uno hace. Incluso hasta los lugares que uno frecuenta, lo que uno hace, si cocina o si no cocina.» (Yesenia)

Lo anterior coincide con lo afirmado por Aimar Christine, Shannon Baird, Precilla Choi, Kerrie Kauer y Vikki Krane, en cuanto a que lo femenino se relaciona con el comportamiento, el cual debe regirse según las normas de etiqueta, ser amable y dulce ${ }^{24}$. Las características que ellas asignan a una mujer limitan el desenvolvimiento libre, porque el comportamiento y la forma de vestir para ellas ya está establecido.

En las entrevistas se evidencia cómo ellas relacionan lo femenino con una condición de género determinada por características dadas a las mujeres, marcadas por ciertos comportamientos y actitudes que para ellas son permitidos o no lo son. Siguen ciertas normas y estereotipos que naturalizan en su cotidianidad y, de alguna manera, las subordina y las transforma en sujetos, ante una construcción social que marca lo que «debe ser» o «no debe ser» una mujer femenina.

Asimismo, ellas describen a una mujer que no es femenina basándose en la apariencia física y el comportamiento. Citan que una apariencia no femenina se refleja al usar ropa floja u holgada, usar cabello corto o desarreglado, tener apariencia descuidada, ser gorda y no usar maquillaje. Entre el comportamiento no femenino mencionan fumar, caminar sin delicadeza, comportamiento brusco y vulgar.

Sus descripciones acerca de una mujer que no es femenina, son las siguientes:

- «Que siempre anda toda desordenada, fea y descuidada (sonríe). Primero en apariencia que no se cuida y que sea gordita, que siempre ande despeinada, no siempre uno anda maquillado, pero por lo menos de vez en cuando maquillarse. Una mujer que no se cuida, que no hace ejercicio.» (Elsa)

- «Es como grosera en su vocabulario, más tirada a hombre que a mujer, su apariencia física no buscan verse como una mujer sino como hombre, usan jeans aguados y camisetas, no usa blusitas, el pelo corto y camina chambón.» (María)

- «Una amiga de mi hija, de femenina no tiene nada, es lesbiana, yo le digo, no puede ser más femenina, es lo que le digo yo, usa zapatos, faja, cadenas, todo como un hombre, es ver un hombre en ella, como camina, es concha y grotesca.» (Ana)

- «Yo digo a veces, esa es como un hombre, porque viste camisetona y zapatos burros o tenis.» (Nancy)

- «Tiene modos muy bruscos y le gusta demostrar que es más fuerte que los hombres.» (Marta)

24. Aimar Christine, Shannon Baird, Precilla Choi, Kerrie Kauer y Vikki Krane, «Living the Paradox: Female Athletes Negotiate Femininity and Muscularity», Revista Sex Roles, n. ${ }^{\circ} 50$ (2004), 315-32. 
- «Usa pantalones, zapato cerrado de cordones, no usa maquillaje, su pelito corto, lentes bien gruesotes, habla vulgar, se sienta con las piernas abiertas.» (Rebeca)

- «Su forma de vestir es parecida a la de un hombre, usa zapato cerrado. La forma de comportarse y de hablar es diferente, usa vocabulario soez y vulgar.» (Yesenia)

- «Que ande con un pantalón todo flojo, la actitud como hombrecillos, que hablen como hombre.» (Sandra)

- «Que no se arregle, que no se preocupe por verse cuidadita.» (Sonia)

- «Se visten como hombre, con unas pantalonetas grandísimas, con tenis que son hasta arriba, con los cordones sueltos, con camisetas grandes, con gorra, hasta usan bóxer de hombre.» (Irma)

- «Grotesca, machorra, que habla vulgar, que camina como hombre, que no se viste bien.» (Patricia)

- «Una mujer que no se cuida, que le da lo mismo tener el pelo feo o bonito, que no se hace nada en las uñas.» (Catia)

Las entrevistadas afirman respetar a las mujeres que no son femeninas, piensan que son así por inseguridad, por problemas en el hogar o por asuntos biológicos. Por ejemplo, Nancy dijo: «Pienso que está mal, porque Dios dejó lo masculino y lo femenino». Yesenia opinó: «Lo respeto pero no lo comparto». Al respecto, Marcela Lagarde se refiere a la falta de feminidad con el término «feminidad fallida»; ella argumenta que la feminidad de las mujeres siempre está a prueba y que las mujeres que transgreden la norma son consideradas mujeres fallidas, sometidas a procesos de descalificación, exclusión y violencia. «Las mujeres deben probar en cada momento de sus vidas su feminidad: si dejan algunos de sus rasgos pierden grados en la valoración social y cultural (...) a partir de una jerarquía consensuada se evalúa por contraste a las mujeres entre sí y frente a los estereotipos (...) de ahí que las mujeres sean calificadas como mejores, buenas, muy malas o peores, en relación a la feminidad» ${ }^{25}$.

Las mujeres entrevistadas tienen miedo de ser ellas quienes pierdan la feminidad; piensan que al adoptar comportamientos y actitudes asignados a roles masculinos podría conducirlas a perder su feminidad y convertirse en el opuesto (hombre masculino), aunque eso no sea realidad, pues que asuman comportamientos del género opuesto no las convierte en hombres. Estos miedos están fundamentados en creencias sociales; ellas tienen miedo a ser señaladas y discriminadas por el incumplimiento de la norma de género y, por eso, adoptan estereotipos femeninos.

\section{Reproducción y mantenimiento de la feminidad}

Todas las mujeres entrevistadas consideran que aprendieron a ser femeninas de la mamá, pues ella les daba ejemplo de cuido de la apariencia, ropa por usar y el maquillaje,

25. Marcela Lagarde y de los Ríos, Los cautiverios de las mujeres: madresposas, monjas, putas, presas y locas (Madrid: Editorial San Cristóbal, Horas y horas, 2011), 786. 
así como en cuanto a la realización de las labores del hogar. Respecto a esto, argumentaron lo siguiente:

- «Mi mamá es súper coqueta.» (Marta)

- «Mi mamá se cuidaba mucho, yo la veía a ella.» (Rebeca)

- «Mi mamá era muy femenina, le gustaba mucho pintarse, le gustaba cocinar.» (Yesenia)

- «Al ver a mi mamá, ella es muy vanidosa.» (Sandra, al narrar la manera como aprendió a ser femenina)

- «Eso viene desde la casa, uno ve a la mamá con las cosas que usa (...) desde pequeña mi mamá me decía que la ropita tenía que ser más al cuerpo.» (Sonia)

- «Mi mamá, ella era muy femenina en su forma de vestir y arreglarse.» (Catia)

- «Mi mamá siempre ha sido muy coqueta, tal vez uno por ver a la mamá. Ella se cuida mucho, siempre anda bonita y tiene buena apariencia.» (Elsa)

Para ellas, la maternidad es determinante en la construcción de la feminidad; es la madre quien enseña a sus hijas cómo deben comportarse y cómo debe ser su apariencia. Además, es ella quien tiene una influencia mayoritaria en su identidad, el solo hecho de ser madre ya es un patrón por continuar.

Todas las entrevistadas consideran que su mamá es o fue femenina. Por ejemplo, Elsa: «A ella le gusta andar bien arregladita, bien pintada, son sus tacones. Ella se cuida mucho». Sandra afirmó: «Ella siempre anda arreglada». Por su parte, Marta aseguró: «Ella le gusta estar siempre arregladita, ella para estar en la casa se pinta los labios, se perfuma, no le gusta salir desarreglada, jamás en sandalias» y Laura comentó: «Ella se arregla lindísima, con ropa alegre, le gusta pintarse y ponerse aretitos».

De las mujeres entrevistadas, cinco dicen que su mamá tiene el cuerpo bonito y que este es delgado (lo catalogan dentro de un parámetro de normalidad por las dimensiones); además, seis dicen que su mamá cuida mucho la apariencia. Al respecto, Sandra mencionó: «Mi mamá es delgada, tiene bonito cuerpo». Mariela citó: «El cuerpo de mi mamá es muy bonito, es delgadito». Laura dijo: «Era de cuerpo bonito, delgado». Por su parte, María aseveró: «Tiene una contextura normal, es delgada». Igualmente, Ana apuntó: «Yo veo el cuerpo de mi mamá bonito y hasta con envidia». En cuanto al cuido, Yesenia afirmó: «Ella dedicaba tiempo a pintarse, todos los días iba a caminar». Asocian la «normalidad corporal» y la belleza a lo delgado.

Ellas mencionan que su mamá les inculcaba ciertas normas de comportamiento, muy relacionadas con los estereotipos de la feminidad. Por ejemplo, María opinó: «Sentarse bien, no hablar, no gritar», mientras Marta indicó que «tenía que cuidar como hablaba» y Yesenia señaló: «Mi mamá me decía que una mujer no tenía que llegar tarde a la casa, ser recatada en sus cosas, no vestirse mal, que tenía que cuidar mi vocabulario».

Mediante estos resultados se comprueba cómo las madres de estas mujeres están o estuvieron perpetuando ideas en torno al género femenino, tal y como lo manifiestan 
Caamaño y Rangel ${ }^{26}$, con el fin de que sus hijas sigan el ideal femenino, por ende, las madres influyeron en la construcción de la feminidad de estas mujeres.

Es, pues, en la familia donde se construyen y promueven los roles de género, entre estos, los femeninos. Este aspecto también es confirmado por León, quien señala que las jerarquías de género son creadas, reproducidas y mantenidas día a día mediante la interacción de los miembros del hogar: «En la familia es donde la división sexual del trabajo, la regulación de la sexualidad y la construcción social y la reproducción de los géneros se encuentra enraizada» ${ }^{27}$.

Estos resultados concuerdan con la teoría de aprendizaje social, de Stassen y Thompson, sobre los principales mecanismos de adquisición de roles femeninos. Las autoras explican que todos los roles de género son aprendidos por medio de la observación, es decir, no son innatos. Indican que la sociedad es la responsable de enseñarlos consciente o inconscientemente y que, además, se dan refuerzos y castigos cuando se comportan de forma en que los adultos consideren apropiada -o inapropiada- según su género ${ }^{28}$.

Las mujeres copian patrones estereotipados sobre la feminidad, estos provienen de observar las acciones y escuchar los discursos de la madre. El lenguaje de la madre ejerce poder sobre sus hijas para constituirlas en sujetos femeninos. Con lo que dicen -a modo de consejo- persuaden a sus hijas; así, las madres manifiestan lo que aceptan y lo que rechazan en ellas mismas al expresar limitaciones, prohibiciones y normas. Los significados que adopten ellas serán primero los significados que tengan sus madres; de esta manera, la madre colabora en la constitución de sus hijas como sujeto femenino.

Sin embargo, las madres responden a toda una estructura social instaurada que se permea en ellas y se reproduce por medio de discursos sociales, puestos en acción en el cuerpo de sus hijas, con la normalización de la dominación y de la subordinación.

Existen dos opciones: reproducir lo aprendido o deconstruir la feminidad. Aunque, por parte de las mujeres, existe un deseo de ser femeninas, como resultado de la interiorización de la cultura dominante y de no caer en el incumplimiento, porque eso podría acarrear señalamiento y rechazo por parte de otras personas; ellas pueden modificar su forma de pensar, construyendo nuevos deseos y formas de vivir. Así como se aprende y construye, se puede desaprender y deconstruir. Para Mandel «la deconstrucción es la estrategia de lectura e interpretación que nos posibilita desarticular y desmontar conceptos fijos, convencionales y normas» ${ }^{29}$. El arte en un medio para lograr esa deconstrucción al trastocar reglas establecidas y propiciar una redefinición del ser mujer.

La imposición de conductas y apariencia femenina genera en las mujeres preocupación e incluso las conduce a buscar maneras de obtener el ideal por medio del disciplinamiento de sus cuerpos con cirugías, dietas, regímenes rigurosos de ejercicio físico y poniendo en riesgo la salud para cumplir con el «deber de ser femenina».

26. Caamaño y Rangel, Maternidad..., 84 .

27. León, La familia..., 180.

28. Stassen y Thompson, Psicología..., 400-405.

29. Mandel, Mapa..., 9. 


\section{Roles de la mujer femenina}

Entre las actividades cotidianas asociadas a la idea de ser mujer que realizan, se encuentran el ser mamá, esposa y hacer todas las labores del hogar. Esto coincide con los resultados del estudio de Teresa Bruel sobre los roles de género, en el cual concluyó que las mujeres se asignan roles de amas de casa y cuido de personas enfermas y de los hijos; por lo tanto, roles de dominio privado y reproductivos, mientras que a los hombres les asignaron roles del dominio de lo público y productivos ${ }^{30}$.

Entre las actividades realizadas diariamente, se encuentra ir al gimnasio, además de las múltiples tareas del hogar. Por ejemplo, Sandra afirmó: "Cocinar, lavar». Laura dijo: «Todo el quehacer de la casa, ser mamá y esposa». Del mismo modo, Yesenia manifestó: «Las cosas de la casa que siempre las hacemos las mujeres, ser esposa y ser madre». De igual forma, Nancy dijo: «Yo hago todo en la casa». Además, María citó: «Limpio, lavo, cocino, atiendo los hijos y a mi esposo, hago compras». También Elsa dijo: «Ser mamá y ama de casa». Ana indicó: «Ser mamá». Igualmente, Patricia agregó: "Cocino, lavo, limpio, veo a mi hijo, estudio y voy al gimnasio». Catia apuntó: «Las normales en una casa» e Irma afirmó: «La casa y cuido los hijos».

Profesionales o no profesionales, todas atienden las tareas de la casa, además de ir al gimnasio para cuidar su apariencia. Al parecer, ir al gimnasio y cuidar la apariencia es tan importante para ellas como atender las labores cotidianas que asignan a una mujer, como los quehaceres domésticos y cuidar de los niños; destacan el rol de cuido en ellas mismas y en los demás.

Es decir, se continúa con la división genérica no solo de comportamientos, sino también de actividades y labores diarias. Ellas consideran que labores como cuidar a los niños y atender el hogar les corresponden y son propias de las mujeres.

\section{Una feminidad para otros}

La mayoría considera que es importante mostrarle su feminidad al esposo, a las hijas (las que las tienen) y una entrevistada respondió que a otras mujeres. Todas se consideran femeninas y afirman esta feminidad frente a los demás mediante su comportamiento, forma de vestir y apariencia.

Aparte de sus labores diarias, estas mujeres dedican tiempo a satisfacer los intereses de sus parejas, para ser sexualmente atractivas y mujeres deseadas, como bien lo manifestó Ana: «Cuando yo me descuidé y tenía 80 kilos, mi esposo no era igual conmigo, de hecho, esa fue la primera vez que nosotros nos separamos, él conoció a otra persona, que aparentemente físicamente es mejor que yo, duramos ocho años separados, después me dio por bajar de peso, y ya no dejaba de ponerme mensajes y después de ahí decidí cuidarme.»

30. Teresa Cristina Bruel dos Santos, «Representaciones sociales de género: un estudio psicosocial acerca de lo masculino y lo femenino» (tesis doctoral, Universidad Autónoma de Madrid, 2008), 174. 
Las entrevistadas piensan que, si su cuerpo fuera diferente, su esposo no estaría con ellas, mientras que seis consideran que a su esposo le importa mucho la apariencia de ellas. En relación con la idea de un cuerpo diferente, Ana dijo: «Si yo hubiera estado gorda ni se hubiera fijado en mí». Además, Laura comentó: «Si yo fuera una mujer gorda gorda, tal vez no». Rebeca detalló: «Si yo fuera gorda mi esposo no estuviera conmigo». Igualmente, Yesenia expresó: «Siendo muy gordilla creo que no estaría conmigo, porque a él le gusta una mujer que se cuide». Con respecto a la apariencia, Ana mencionó: «Él dice que le gusta como me cuido, que soy demasiado vanidosa». También Nancy dijo: «Por ejemplo, ayer en la noche hice picadillo prensado con arroz y frijoles, me dice: 'Diay, pero se va a engordar'». Igualmente, Marta indicó: «Me dice que le ponga en el gimnasio», y Laura mencionó: «No le gusta que sea gorda».

Además, afirman que sus parejas desaprueban ciertos comportamientos en una mujer. Elsa comentó: «Que se descuiden». Por su parte, María: «Que usen un vocabulario vulgar, para él es muy desagradable ver a una mujer borracha y por tanto los comportamientos que asumen cuando están así, sobre todo cuando están con hombres». De igual manera, Laura señaló: «No le gusta la mujer mal hablada o que fume». Además, Rebeca dijo: «Él no soporta la gente desordenada, toda sucia, sudada, en chancletas, con ropa fuera de lugar, vulgar, el comportamiento y la educación de una mujer le importan» y Marta afirmó: «La voz alta y la impulsividad».

Todos estos resultados concuerdan con los obtenidos por Adelina Calvo, Susana Rojas y Teresa Susinos, quienes concluyeron que la feminidad puede ser extrapolada no solo por la imagen que las mujeres tienen de sí mismas, sino por el relato de sus vidas y por las valoraciones de otras personas que forman parte de sus vidas (familiares, pareja) ${ }^{31}$.

\section{Conclusiones}

¿Cuál es el significado que dan las mujeres que realizan AFD a la feminidad? A esta pregunta, se concluye:

- El concepto de feminidad tiene una vinculación directa con el cuerpo de las mujeres.

- El significado de la feminidad proviene de dos factores: apariencia y comportamiento, ambos relacionados con estereotipos que limitan el libre desenvolvimiento de la mujer.

- El significado de feminidad de las entrevistadas es concordante con lo externo de las mujeres.

- Se comprueba que lo femenino es un ideal que norma el comportamiento de las mujeres con roles que dictan actitudes pasivas al hablar y caminar, como lo expresaron las entrevistadas.

31. Adelina Calvo, Susana Rojas \& Teresa Susinos, «Becoming a woman: the construction of female subjectivities and its relationship with school», Gender and Education, n. ${ }^{\circ} 2$ (2009), 97-110. 
- La constitución de la feminidad es resultado tanto del medio familiar como del social, al recibir las representaciones de la feminidad de la familia y de la sociedad y luego las proyectan externamente.

- La construcción de la feminidad está basada en los estereotipos de la apariencia y del ideal corporal para las mujeres, en los roles asignados a ellas, como el de la maternidad y la heterosexualidad, así como estereotipos sobre el comportamiento y desempeño de la mujer en sociedad. También, ejerce una fuerte influencia en esa forma de construirse la relación con la pareja y la necesidad de cumplir con los requerimientos de lo que se «debe ser» para satisfacerlo.

- La feminidad es pensada desde una construcción social subjetiva que viene de lo que reciben del entorno hasta operacionalizarlo en su cuerpo. De manera que el cuerpo de la mujer es centro para manifestar la construcción de la feminidad.

- La feminidad es un fenómeno que responde a la intersubjetividad, porque las mujeres construyen esa feminidad subjetivamente en interacción con «otros», primordialmente por la influencia de la madre.

- Las mujeres siguen las normas de la feminidad porque temen ser juzgadas por los otros, principalmente su madre y pareja, temen ser juzgadas por causa de su apariencia física y su comportamiento. Son los otros quienes dan lectura a su apariencia, aceptándola o rechazándola.

- La feminidad es adquirida por ser seguir modelos establecidos socialmente como parte de un proceso de socialización que inicia en la niñez, cuando adoptaron roles y actitudes de los adultos a su alrededor, hasta apropiárselos.

\section{Reflexión final}

La visibilización de temáticas como las tratadas en esta investigación, permite, de alguna manera, el cuestionamiento de la construcción subjetiva de la feminidad. Se debe considerar que las subjetividades femeninas responden a una cultura androcéntrica, en la cual se presentan intereses de poder mediados por el afán de mantener el dominio sobre el «otro» desde estereotipos que son reproducidos y mantenidos por diferentes medios.

La feminidad, como subordinación, limita de alguna manera el desenvolvimiento pleno de las mujeres, limita la posibilidad de escoger su apariencia, comportamiento y manera de vivir. Las mujeres se preocupan ante el posible incumplimiento del ideal que genera temor al señalamiento, crítica, anulación, rechazo, discriminación y exclusión. Miedo a perder el lugar en el género femenino y ser descalificadas.

Ser mujeres en una estructura androcéntrica dominante y normativa no quiere decir que no pueda construirse de una manera diferente, partiendo de una reformulación de pensamiento y vivencias corporales en inclusión universal, dando significados diferentes a lo femenino.

En la actualidad, varias mujeres han salido de la homogenización y estandarización de su ser y han alzado públicamente la voz por otras mujeres, así como la resignificación 
de sus cuerpos ante la regulación de los mismos. Esto da valor a otras mujeres para salir de sus cautiverios, los cuales son variados.

Por lo tanto, los estereotipos normativos frente a la feminidad no son más que subordinación y dominación hacia las mujeres. Ante esto surgen interrogantes, principalmente sobre cuáles son los intereses de la sociedad patriarcal en que las mujeres sean sumisas, débiles, pasivas e irracionales.

\section{ABSTRACT}

\section{The meaning of femininity: study based on life stories of women}

With the purpose of determining the meaning that the characteristics and the roles attributed to women give to femininity, 14 women were interviewed. Based on the participants' answers, it is determined that the meaning of femininity derives from two factors: appearance and behaviour. Having that in mind, it can be concluded that femininity has a direct link to women's bodies and is based on ideals, stereotypes, roles and norms assigned by the patriarchal structure which have been established and have permeated the corporeal, from the way of thinking to the replication of behaviors that consequently impact women's lives. Therefore, the model of femininity is socialized by a whole structure to be established, reproduced and maintained.

Key words: Gender, construction, appearance, behavior.

\section{RÉSUMÉ}

\section{Le sens de la féminité: étude basée sur les histoires de vie de femmes}

Afin de déterminer la signification de la féminité à partir des caractéristiques et des rôles attribués aux femmes, on a interviewé quatorze femmes. Ceci a permis de comprendre que le sens de la féminité provient de deux facteurs : l'apparence et le comportement. En outre, on a pu conclure que la féminité est liée avec le corps de la femme et qui est basée sur les idéaux, les stéréotypes, les rôles et les normes établis par la structure patriarcale. Ces derniers se sont instaurés et ont imprégné les corporalités ; dès la manière de penser jusqu'à la reproduction de comportements et cela a impacté la vie des femmes. C'est ainsi que le modèle de féminité a été socialisé par une structure dans le but d'être instauré, reproduit et conservé.

Mots-clés: Genre, construction, apparence, comportement.

\section{Referencias bibliográficas}

Aimar Christine, Shannon Baird, Precilla Choi, Kerrie Kauer y Vikki Krane. «Living the Paradox: Female Athletes Negotiate Femininity and Muscularity». Sex Roles, n. ${ }^{\circ} 50$ (2004): 315-329.

Amorós, Celia. Feminismo y filosofía. Madrid: Editorial Síntesis, 2000.

Bautista, Juan. El laberinto de la feminidad y el acto analitico. Buenos Aires: Homo Sapiens, 2009.

Butler, Judith. Deshacer el género. El género en disputa: el feminismo y la subversión de la identidad. Barcelona: Paidós, 2006. 
Bruel dos Santos, Teresa Cristina. «Representaciones sociales de género: un estudio psicosocial acerca de lo masculino y lo femenino». Tesis doctoral. Universidad Autónoma de Madrid, España, 2008.

Caamaño, Carmen y Ana Rangel. Maternidad, feminidad y muerte. San José: Editorial Universidad de Costa Rica, 2002.

Calvo Adelina, Rojas Susana \& Teresa Susinos. «Becoming a woman: the construction of female subjectivities and its relationship with school». Gender and Education, n. ${ }^{\circ} 2$ (2009): 97-110.

Fernández Carvajal, Doris. La construcción de la subjetividad de las mujeres en una sociedad costera. Revista PRAXIS, n. ${ }^{\circ} 60$ (2007): 29-48.

Lagarde y de los Ríos, Marcela. Los cautiverios de las mujeres: madresposas, monjas, putas, presas y locas. Madrid: Editorial San Cristóbal, Horas y horas, 2011.

León, Magdalena. «La familia nuclear: origen de las identidades hegemónicas femenina y masculina». En Género e identidad: ensayos sobre lo femenino y lo masculino, editado por Gabriela Arango, Magdalena León y Mará Vivero. Ediciones Uniandes, 1995.

Mandel, Claudia. Mapa del cuerpo femenino. Una lectura deconstructiva de creadoras visuales en Costa Rica. Costa Rica: Editorial UCR, 2010.

Mayobre Rodríguez, Purificación. «La formación de la identidad de género. Una mirada desde la filosofía». Revista venezolana de estudios de la mujer, $\mathrm{n}^{\circ} 12$ (2006): 35-62. Acceso: 03 de marzo, 2020. http://www. scielo.org.ve/scielo.php?script=sci_arttext\&pid=S1316-37012007000100004\&lng=en\&nrm=iso\&tlng=es

Sanahuja YII, María Encarna. Cuerpos sexuados, objetos y prehistoria. Madrid: Ediciones Cátedra, 2002.

Santillán, E. M. Mujer, deporte y fútbol: una perspectiva de género. Distrito Federal, 2006.

Segato Laura, Rita. «Las nuevas formas de la guerra y el cuerpo de las mujeres». Revista Sociedade e Estado, n. ${ }^{\circ} 29$ (2014): $341-371$.

Stassen, Kathlenn, y Ross Thompson. Psicología del desarrollo. Infancia y adolescencia. Madrid: Editorial Médica Panamericana, 1997.

Tubert, Silvia. «Los ideales culturales de la feminidad y sus efectos sobre el cuerpo de las mujeres». Quaderns de Psicología, n. ${ }^{\circ} 12$ (2010):161-174. Acceso: 03 de marzo, 2020. http://www.quadernsdepsicologia.cat/ article/view/7602010

Venegas Medina, María del Mar. «La "mirada normativa del otro", representación del cuerpo femenino y construcción de la identidad corporal a través de la experiencia del cuerpo como espacio de sumisión y resistencia». En Cuerpos de mujeres: miradas, representaciones e identidades, editado por Gregorio Gil, Carmen, Muñoz Muñoz, Ana y Sánchez Espinosa Adelina, Granada: Editorial Universidad de Granada, 2007.

Vera, Celia. Género, cultura e imagen del cuerpo. En Derecho de las mujeres a su imagen: los trastornos del comportamiento alimentario (anorexia y bulimia). Universidad de Alicante, 2001. 